\title{
Rapid measurement of intravoxel incoherent motion (IVIM) derived perfusion fraction for clinical magnetic resonance imaging
}

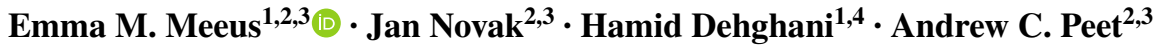

Received: 24 June 2017 / Revised: 26 September 2017 / Accepted: 27 September 2017 / Published online: 26 October 2017

(C) The Author(s) 2017. This article is an open access publication

\begin{abstract}
Objective This study aimed to investigate the reliability of intravoxel incoherent motion (IVIM) model derived parameters $D$ and $f$ and their dependence on $b$ value distributions with a rapid three $b$ value acquisition protocol.

Materials and methods Diffusion models for brain, kidney, and liver were assessed for bias, error, and reproducibility for the estimated IVIM parameters using $b$ values 0 and 1000, and a $b$ value between 200 and 900, at signalto-noise ratios (SNR) 40, 55, and 80. Relative errors were used to estimate optimal $b$ value distributions for each tissue scenario. Sixteen volunteers underwent brain DW-MRI, for which bias and coefficient of variation were determined in the grey matter.

Results Bias had a large influence in the estimation of $D$ and $f$ for the low-perfused brain model, particularly at lower $b$ values, with the same trends being confirmed by in vivo imaging. Significant differences were demonstrated in vivo for estimation of $D(P=0.029)$ and $f(P<0.001)$ with $[300,1000]$ and $[500,1000]$ distributions. The effect of bias was considerably lower for the high-perfused models.
\end{abstract}

Andrew C. Peet

a.peet@bham.ac.uk

1 Physical Sciences of Imaging in Biomedical Sciences (PSIBS) Doctoral Training Centre, University of Birmingham, Birmingham B15 2TT, UK

2 Institute of Cancer and Genomic Sciences, University of Birmingham, Birmingham B15 2TT, UK

3 Department of Oncology, Birmingham Children's Hospital, Steelhouse Lane, Birmingham B4 6NH, UK

4 School of Computer Science, University of Birmingham, Birmingham B15 2TT, UK
The optimal $b$ value distributions were estimated to be brain $_{500,1000}$, kidney 300,1000 , and liver 200,1000 .

Conclusion IVIM parameters can be estimated using a rapid DW-MRI protocol, where the optimal $b$ value distribution depends on tissue characteristics and compromise between bias and variability.

Keywords Diffusion weighted magnetic resonance imaging (DW-MRI) · Biological models · Perfusion · Intravoxel incoherent motion (IVIM)

\section{Introduction}

The acquisition of multi- $b$ value diffusion-weighted magnetic resonance (DW-MRI) data and the bi-exponential signal decay observed in biological tissue have led to an increased number of studies using the intravoxel incoherent motion (IVIM) model [1, 2]. The IVIM model can be used to investigate the underlying tissue microenvironment and is based on the simultaneous assessment of two diffusion components. These correspond to the molecular diffusion in tissue $(D)$ and diffusion affected by perfusion in the microcapillary network, often described as pseudo-diffusion $\left(D^{*}\right)$. The model also determines the fraction of signal arising from the microvascular network $(f)$, known as perfusion fraction, which is thought to describe the vascularity of the tissue [1]. The IVIM model parameters have shown clinical value in the imaging of many different tumour types [3-5], as well as stroke $[6,7]$ and liver cirrhosis $[8,9]$. The use of multi- $b$ value DW-MRI has the potential to provide a single acquisition protocol for the non-invasive assessment of diffusion and perfusion in tissue.

The clinical adoption of the IVIM model has been hindered by practical issues and lack of consensus such as 
the number and choice of $b$ values and requirements for a sufficient signal-to-noise ratio (SNR) level for accurate and reproducible post-processing [10-12]. Previous studies have demonstrated promising reproducibility for IVIM parameters $D$ and $f[3,13]$, including a multi-centre brain study [14], whereas greater variability has been shown for the $D^{*}$ parameter. The application of the IVIM model has been well established in abdominal organs such as liver [11] and kidney [15], but applications in the brain [4, 16] have been more challenging due to the relatively low perfusion. However, recent studies have suggested that the use of the IVIM model, and the $D$ and $f$ parameters, has potential in brain tumour grading $[4,17]$ and stroke imaging $[6,18]$. While these studies imply the clinical value of IVIM in the brain, the reported $f$ values have been inconclusive with high variability, potentially owing to the different $b$ value distributions used in the data acquisitions.

The translation of IVIM to clinical practice requires a DW-MRI protocol with a short acquisition time. One way to reduce the scanning time is to decrease the number of $b$ values. Previously, a constrained (also known as segmented) IVIM fitting approach has been shown to provide the most robust IVIM parameters in many tissue types $[10,13,19]$. Using this methodology, the $D$ and $f$ parameters can be computed using high $b$ values and $b=0 \mathrm{~s} / \mathrm{mm}^{2}$. Additional low $b$ values are required for the computation of the $D^{*}$ parameter. However, the challenges with the accuracy and reproducibility of $D^{*}$ in both brain and body, suggest that further evaluation is required to demonstrate its clinical value in terms of its reliability $[12,19-21]$. Therefore, if only $D$ and $f$ are of clinical interest, the IVIM could be performed using a set of high $b$ values, thereby minimising the time required for data acquisition.

The constrained fitting was recently used in a study by Conklin et al. [18], where the IVIM $f$ parameter was estimated with a series of high $b$ value combinations for brain tumour and stroke patients. The recommended $b$ value distribution was chosen by comparison to the more commonly used fitting method (2-parameter fitting method [13]) in the brain. Although the similarity of the two fitting methods can indicate how many $b$ values are required for comparable results, it is unable to assess the accuracy of the estimated IVIM parameters. Therefore, the purpose of this study was to use a minimum number of $b$ values to minimise the scan duration and to assess the reliability of the estimated $D$ and $f$ parameters with different $b$ value distributions, using simulated models with known ground truth values, and compare these results to IVIM data collected in vivo.

\section{Materials and methods}

\section{Data simulations}

All simulations and data analysis were implemented in MATLAB (MathWorks, Natick, MA, USA, v.2016b). The model data signal values were generated with Eq. 1 using a $b$ value distribution: $0,200,300,400,500,600,700,800$, 900, $1000 \mathrm{~s} / \mathrm{mm}^{2}$, as described in Fig. 1.

$S(b) / S(0)=f \cdot \exp \left(-b D^{*}\right)+(1-f) \cdot \exp (-b D)$

Signal data sets were generated using a range of $f$ values (0.06-0.30 in increments of 0.02) and three different $D^{*} / D$ ratios corresponding to previously reported ratios observed in the brain, kidney and liver $(10,20$, and 70 , respectively) [12]. The $D$ parameter was fixed at $0.7 \times 10^{-3} \mathrm{~mm}^{2} / \mathrm{s}$ and $D^{*}$ parameters used were brain: $7 \times 10^{-3}$, kidney: $14 \times 10^{-3}$ and liver $49 \times 10^{-3} \mathrm{~mm}^{2} / \mathrm{s}$. In this study, these models are also referred to as low-, medium-, and highperfusion models, respectively.

Signal data for the different diffusion models and a series of $f$ values is presented in Fig. 1b, c. Gaussian noise was introduced to the modelled data to simulate the Rician distribution of noise found in MR images using the in-built MATLAB function (Communications System toolbox). The Gaussian approximation is sufficient for the signal-to-noise ratio (SNR) levels of 40,55 , and 80 , which were used to study the influence of noise on the estimated parameters [22]. SNR $=40$ was chosen based on previous on-site measurements of diffusion-weighted MRI data [13] and the in vivo data presented here. The higher SNR levels 55 and 80 corresponded to approximately increasing the number of signal averages (NSA) from one to two and four, respectively. The same SNR level was used for all data points at different $b$ values. The data simulations were performed using $N=1,000$ random data iterations for each set of IVIM parameters.

\section{Volunteer population}

A cohort of healthy young adult volunteers $(n=16$, age $25-30$, mean age 26 years) was scanned using a multi- $b$ value diffusion-weighted imaging and $T_{1}$-weighted imaging protocols. The protocols for this retrospective study were approved by the East Midlands - Derby Research Ethics Committee (REC 04/MRE04/41) operating under the rules of Declaration of Helsinki 1975 (and as revised in 1983), and informed consent was obtained from all volunteers. 
(a)

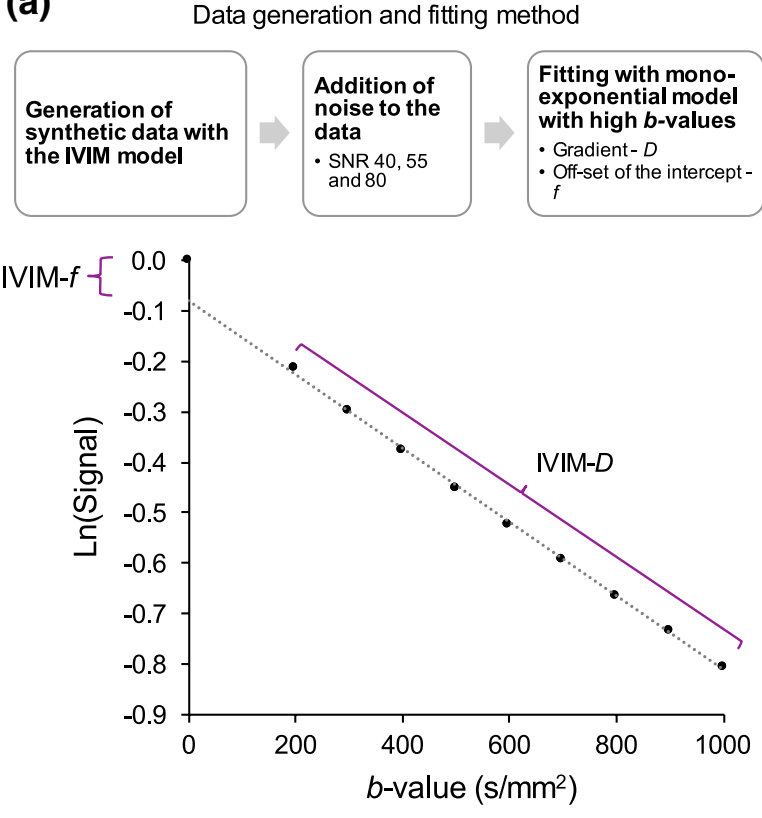

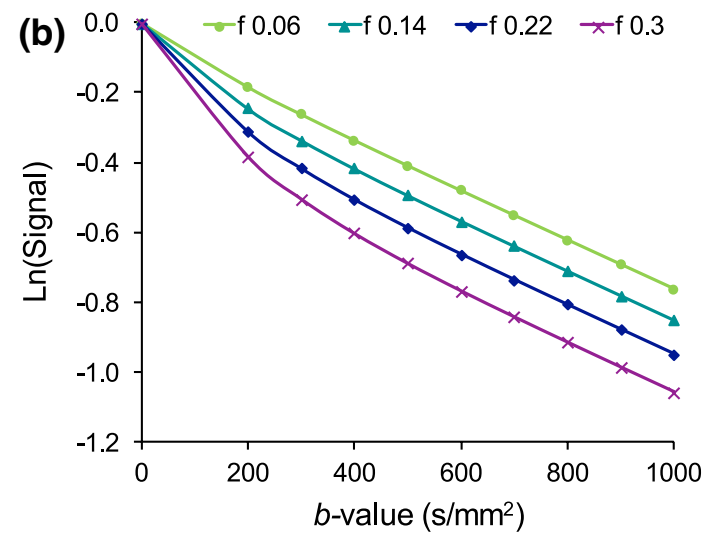

(c)

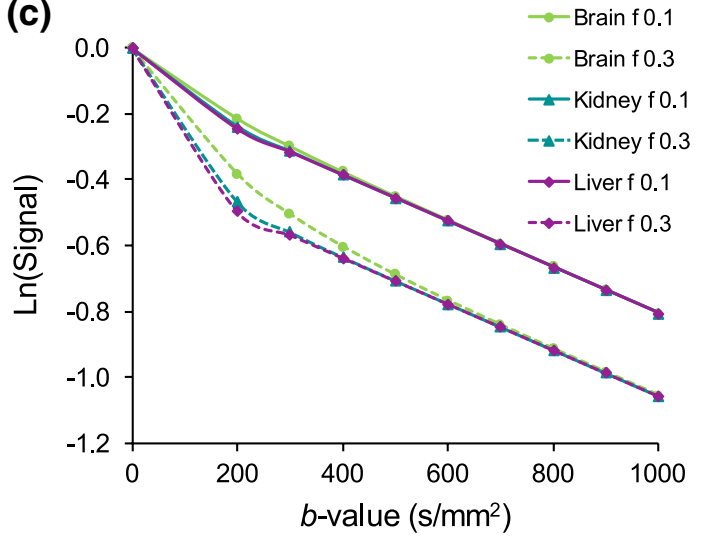

respectively, and $\mathbf{b}$ the data signal decay at varying $f$ values for the low-perfusion model (brain) and comparison of the signals for different perfusion scenarios, respectively

Additionally, four of the volunteer cases $(n=4)$ were scanned twice with the above DW-MRI protocol to assess the IVIM parameter repeatability.

\section{Data analysis}

The data fitting was performed with the previously [13] reported constrained fitting method, shown in Fig. 1a. The fitting of the simulated diffusion-weighted signal was performed with $b$ value distributions: [200,1000], [300,1000], [400,1000], [500,1000], [600,1000], [700,1000], [800,1000], and $[900,1000]$. Using the assumption that no IVIM effect is observed at high $b$ values [23], the method allows the computation of $D$ and $f$ using the mono-exponential equation:

$S(b) / S(0)=\exp (-b D)$

The $f$ can be measured from the mono-exponential fit by extrapolating it to the $y$-intercept $S$ (int) and taking the difference to the signal from $S(0)$ :

$f=1-S($ int $) / S(0)$ with a spin-echo sequence with FOV $240 \times 240 \mathrm{~mm}$, matrix size $240 \times 240$, slice thickness $3.5 \mathrm{~mm}$ and TR/ $\mathrm{TE}=675 / 10 \mathrm{~ms}$. 
The in vivo DW-MRI data $(n=16)$ was fitted using $b$ value distributions $[300,1000]$ and $[500,1000]$, including the scans acquired for repeatability measurements. SNR levels of the data were determined using the standard NEMA method based on a difference image of two acquisitions, which is the recommended method for computing SNR when parallel imaging such as SENSE acceleration is used [24]. The SNR at $b=1000 \mathrm{~s} / \mathrm{mm}^{2}$ was found to be in the range of $45 \pm 8$ and was similar across the brain.

The in vivo grey matter masks were created for each volunteer case with the brain extraction tool (BET) and FMRIB's automated segmentation tool (FAST) in FMRIB Software Library package (Analysis Group, FMRIB, Oxford, UK, v. 5.0) using the $T_{1}$-weighted images [25, 26]. The probabilistic tissue segmentation was performed for three classes, corresponding to grey matter, white matter, and cerebrospinal fluid (CSF). To assess the inclusion of only cortical grey matter and exclusion of sulcal CSF in the binary masks, partial volume tissue (PVE) segmentation was also performed for eight of the volunteer cases $(n=8)$. The PVE masks provided an estimation of the proportion of grey matter within the voxels (scale $0-1$ ), and only voxels of value $=1$ were included in the analysis, which corresponded to tissue fully representing grey matter with no partial volume of CSF or white matter. The $T_{1}$-weighted images were acquired using the same spatial geometry as the DWI images, and both were visually inspected for any distortions. No further registration was performed at postprocessing. The masks were adjusted for the size of the acquired DWI images using bi-linear interpolation, and a threshold of $T=0.7$ was applied to remove any blurring effects around the edges. This further minimised the number of pixels affected by partial volume effects. For the analysis, the IVIM $D$ and $f$ values were extracted using the grey matter masks from three slices above the lateral ventricles.

Based on the extracted grey matter values, average histograms were computed for the IVIM parameters. The number of bins was based on the square root of the maximum number of data values extracted from the regions-of-interests (ROIs). The bin widths were computed for a range of zero to the maximum IVIM value. The same number of bins was used for all the cases and $b$ value distributions, as well as for the IVIM values extracted with the PVE masks.

The same histogram methodology was applied to the simulated IVIM parameters.

The artwork in this manuscript was created with Microsoft Excel (Microsoft, Redmond, WA, USA, v.16.0) and Inkscape (GNU General Public Licence, v.0.91).

\section{Statistical analysis}

All statistical analysis was performed in SPSS Statistics (IBM, Chicago, IL, USA, v.22). The following statistics were calculated for the data simulations and the estimated $D$ and $f$ parameters. Relative bias was determined from the difference between the true parameter (used in signal data generation) and the estimated parameter (computed from fitting of the signal data), which was normalised to the true parameter value:

Relative bias $=\frac{\frac{1}{N} \sum_{i=1}^{N}\left(x_{i}-X\right)}{X}$

where $i=$ number of iterations, $x_{\mathrm{i}}=$ estimated parameter and $X=$ true parameter. Relative error $(\sigma)$ was computed as the root mean square of the distance between the true parameter to the estimated parameter:

Relative error, $\sigma=\frac{\sqrt{\frac{1}{N} \sum_{i=1}^{N}\left(x_{i}-X\right)^{2}}}{X}$

Both relative bias and error were determined individually for each estimated parameter $(D, f)$ rather than for the mean values over all data iterations. The overall relative error was computed from the individual parameter errors for each $b$ value distribution using $\sigma_{D+f}=\sigma_{D}+\sigma_{f}$. The overall error was used to make recommendations for the simulated tissue regions based on the smallest overall relative error.

The reproducibility of the estimated parameters was determined as a coefficient of variation from the ratio of the standard deviation to the mean of the estimated parameters:

Coefficient of variation $(\%)=\left(\frac{\sqrt{\frac{1}{N} \sum_{i=1}^{N}\left(x_{i}-\bar{x}\right)^{2}}}{\bar{x}}\right) \times 100$

where $\bar{x}$ is the mean of the estimated parameter $D$ or $f$.

For the in vivo data $(n=16)$, correlation analysis (Pearson correlation coefficient, $r$ ) was performed for the mean IVIM parameters in grey matter, to determine how the values were related between the $b$ value distributions [300,1000] and [500,1000]. An analysis of variance (ANOVA) was performed to test if the estimated parameters differed significantly $(P<0.05)$. Bland-Altman analysis was used to determine the bias between the $b$ value distributions. The repeatability of the IVIM parameters was tested using within-subject coefficient of variation $\left({ }_{w} \mathrm{CV} \%\right)$, which was the recommended statistic by the quantitative imaging biomarkers alliance [27] and has been applied in previous studies $[3,11,19]$. The ${ }_{w} \mathrm{CV}$ was computed with the root mean square method [28], using the paired DW-MRI data measurements $(n=4)$ and $4 \times 4$ ROIs (two from each measurement pair) of the same grey matter regions as used in the above analysis. To assess whether the IVIM values were influenced by CSF partial volume, ANOVA was performed 
for the IVIM histogram parameters derived with the probabilistic and PVE masks $(n=8)$ to determine any significant difference $(P>0.05)$.

\section{Results}

\section{Model data}

The relative bias results for the estimated $D$ and $f$ parameters from the low-, medium-, and high-perfusion tissue models are presented in Fig. 2 for the different $b$ value distributions and noise levels. Noise was found to influence the bias at $\mathrm{SNR}=40$ for high $b$ values, whereas results at SNR $=55$ and 80 resembled one another in magnitude and behaviour for all tissue models. The direction of bias was different for
$D$ and $f$, with positive and negative bias shown, respectively. At the higher SNR levels (55 and 80), the magnitude of the simulated $f$ value was found to not affect the bias in estimation of $f$. However, at SNR $=40$, noise influenced the simulated $f$ values to a different extent at higher $b$ values. The similarity of biases at $\mathrm{SNR}=55$ and 80 , suggest that these present the intrinsic magnitude of biases from the fitting of the tissue models. Higher biases were observed for the lower perfusion models with lower $D^{*} / D$ ratio equating to lower degree of bi-exponential behaviour.

The choice of $b$ value had a noticeable influence on the observed bias. The intrinsic bias of the models and estimated parameters was higher at low $b$ value distributions, whereas noise affected the high $b$ value distributions, although only for the $f$ parameter. The bias of $f$ for the low-perfusion model at SNR $=40$ was $-21.6 \pm 0.27,-8.63 \pm 0.8$, and
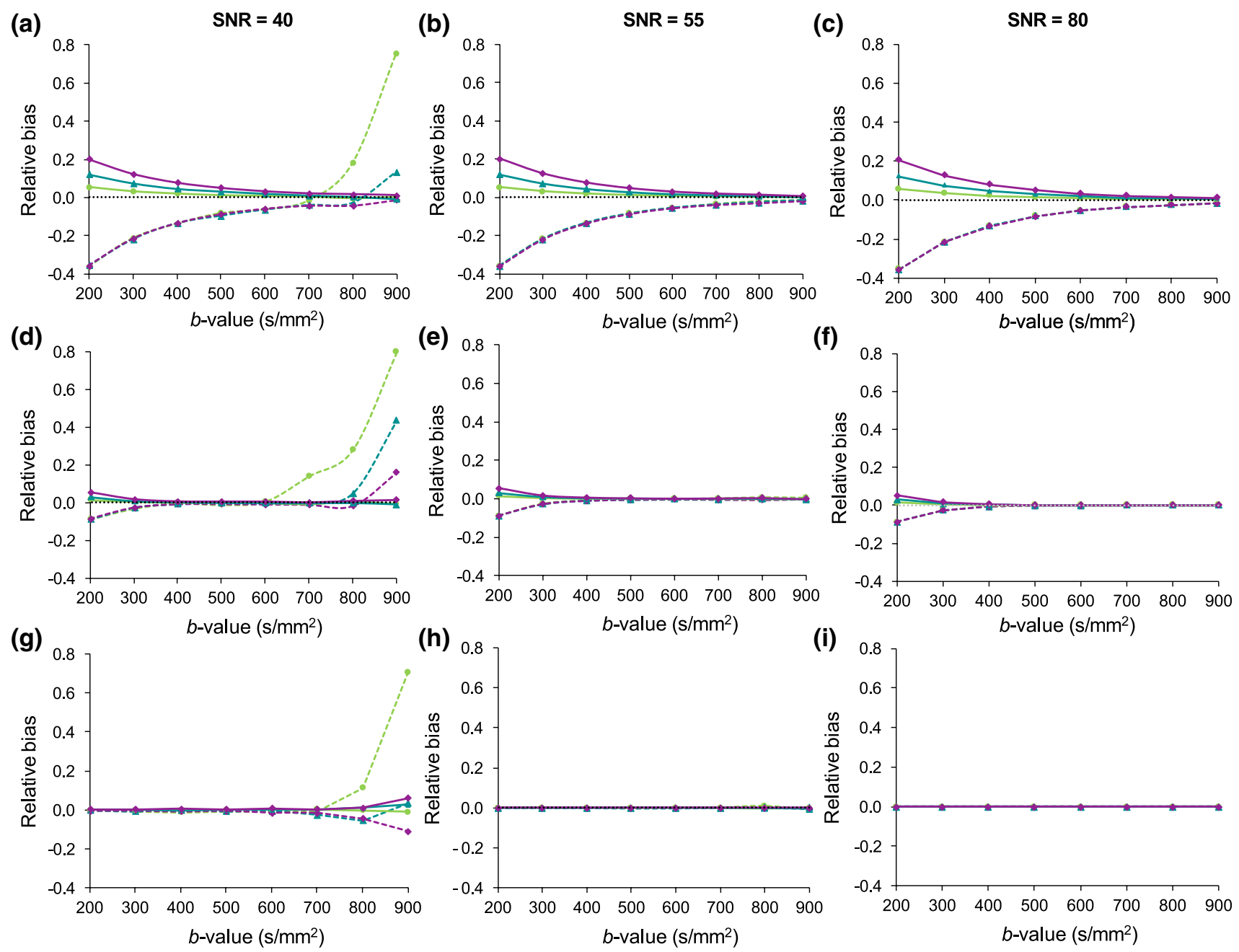

$$
\rightarrow f=0.1 \rightarrow-\oplus f=0.2 \rightarrow f=0.3 \rightarrow D \text { at } f=0.1 \rightarrow D \text { at } f=0.2 \rightarrow D \text { at } f=0.3
$$

Fig. 2 Relative bias results for a-c low-, d-f medium-, and $\mathbf{g}-\mathbf{i}$ high-perfusion models at SNR levels 40, 55, and 80 as a function of $b$ value. Results are presented for simulated $f$ values of $0.1,0.2$, and 0.3 for both $D$ and $f$. Bias $=0$ is indicated by the black dashed line 
$-5.77 \pm 15.0 \%$ for $[300,1000],[500,1000]$, and $[800,1000]$ distributions, respectively. At SNR $=55$ the biases were similar, but with reduced variability: $-21.7 \pm 0.07,8.48 \pm 0.05$, and $-2.62 \pm 0.32 \%$. Similarly for the $D$ parameter at $\mathrm{SNR}=40$, the bias was $6.82 \pm 3.31 \%, 2.72 \pm 1.34 \%$, and $0.61 \pm 0.65 \%$ for $[300,1000]$, [500,1000], and $[800,1000]$ distributions, respectively. For the higher perfusion models, the bias was $<10 \%$ for $f$, apart from the high $b$ value distributions ( $\left.b=700-900 \mathrm{~s} / \mathrm{mm}^{2}\right)$ at SNR $=40$. The bias for the $D$ parameter was $<6 \%$ for both higher perfusion models.

The reproducibility results for $D$ and $f$ parameters and the different tissue models are presented in Figs. 3 and 4, respectively. The variability of the estimated IVIM parameters was largely influenced by noise and dependent on the SNR level. The increase from SNR $=40$ to $\mathrm{SNR}=55(\mathrm{NSA}=1$ to NSA $=2$ ) resulted in a noticeable improvement in the reproducibility of $D$ and $f$, with a smaller improvement observed with the increase to SNR $=80$. The coefficient of variation (\%) of $f$ for the low-perfusion model at SNR $=40$ was $12.4 \pm 7.4 \%, 17.6 \pm 10.0$, and $41.7 \pm 12.6 \%$ for $[300,1000]$, $[500,1000]$, and $[800,1000]$ distributions, respectively. At $\mathrm{SNR}=55$ these were reduced to: $2.23 \pm 1.34,3.14 \pm 1.88$, and $9.83 \pm 6.04 \%$. The different tissue models did not differ to a great extent in terms of their reproducibility for the $f$ parameter, but the $D$ parameter was found to be more reproducible with the low-perfusion model. Lower variability of $D$ and $f$ was observed with the use of lower $b$ value distributions and the higher $f$ values had better reproducibility compared to the low $f$ values.

The overall relative errors based on both $D$ and $f$ errors are summarised in Table 1 and presented visually in Fig. 5 for the $f$ parameter. The overall error was largely influenced by the relative error of $f$ with small contribution from the relative error of $D$. The relative error of $f$ was greater than $D$ in all cases. At SNR $=80$ for low- and medium-perfusion models, the relative errors were higher at low $b$ value distributions because of the bias, whereas negligible bias was observed with the high-perfusion model. At SNR $=40$, noise had a larger influence on the estimated values compared to bias, resulting in higher relative errors at high $b$ values. At $\mathrm{SNR}=55$, similar magnitude of contribution from bias and noise were seen for the low-perfusion model, whereas noise was the dominant contributor for the higher perfusion models.

Based on the minimal overall and $f$ relative errors, suggestions for optimal $b$ value distributions were derived.
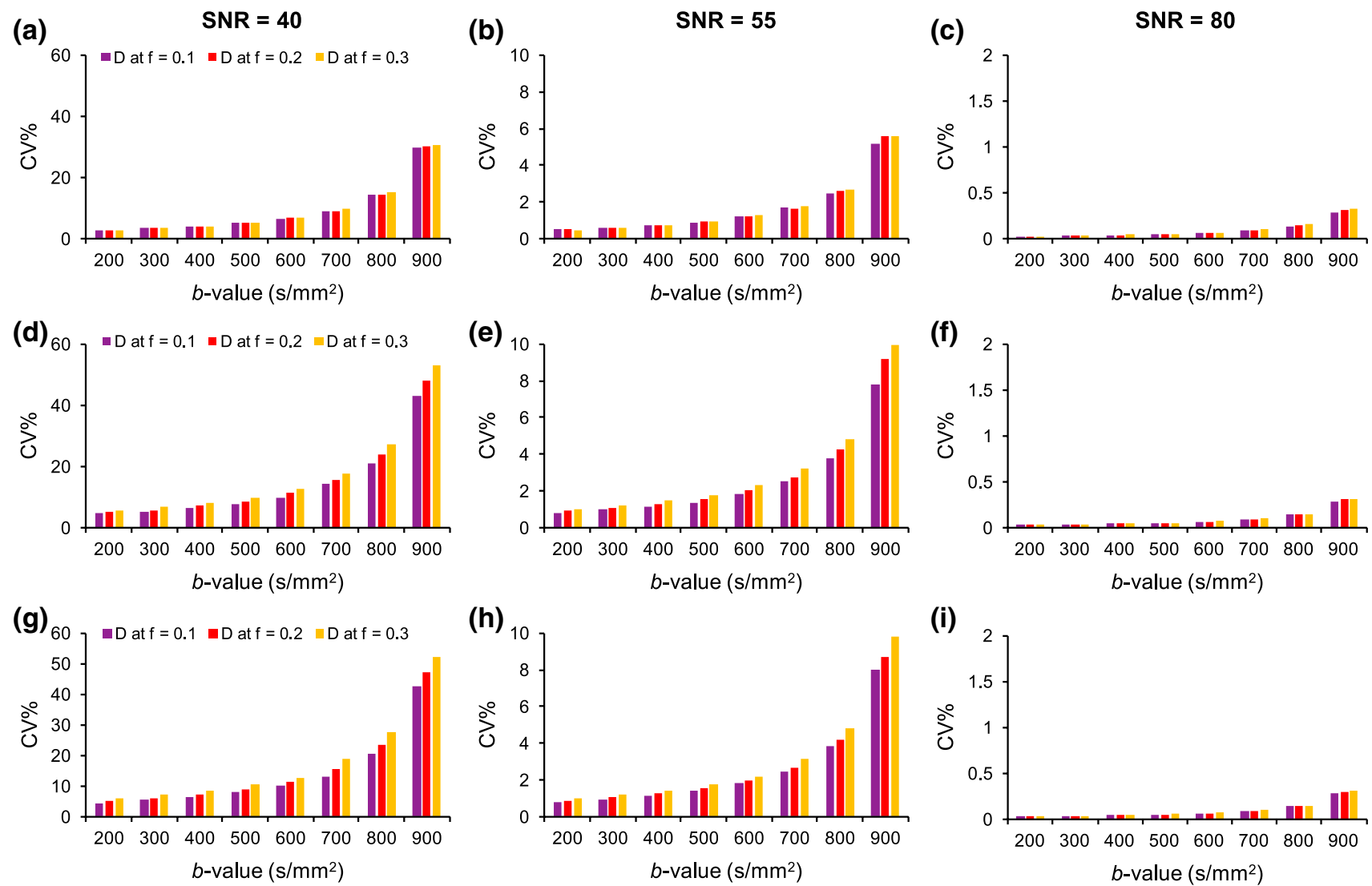

Fig. 3 Reproducibility of diffusion coefficient, $D$, in low- $(\mathbf{a}-\mathbf{c})$, medium- $(\mathbf{d}-\mathbf{f})$, and high-perfusion (g-i) models at SNR levels 40 , 55, and 80 for simulated $f$ values: $0.1,0.2$, and 0.3 

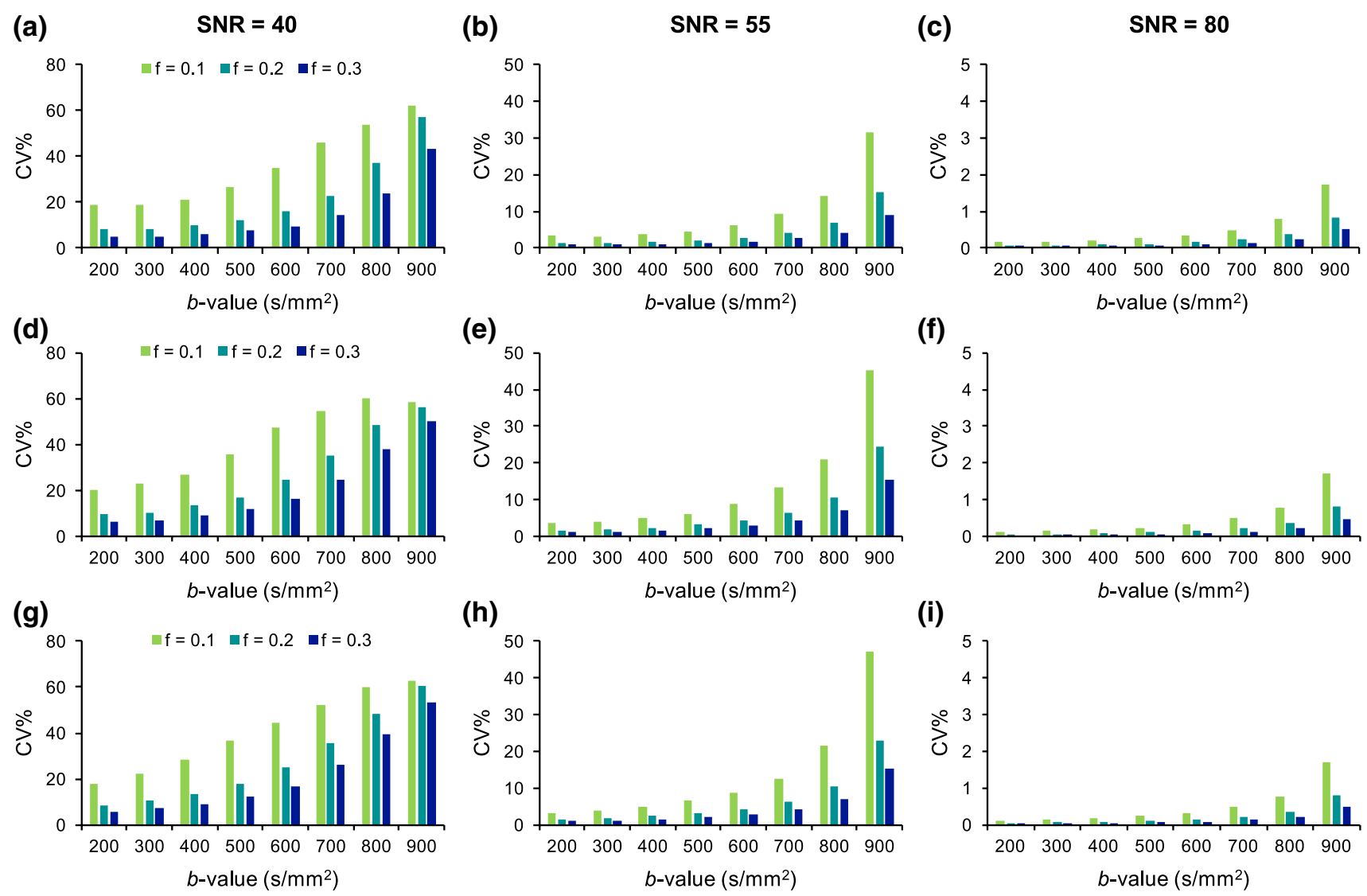

Fig. 4 Reproducibility of perfusion fraction, $f$, in low- $(\mathbf{a}-\mathbf{c})$, medium- $(\mathbf{d}-\mathbf{f})$, and high-perfusion (g-i) models at SNR levels 40,55 , and 80 as a function of $b$-value for simulated $f$ values: $0.1,0.2$, and 0.3

Table 1 Overall relative error ( \pm standard deviation) of the estimated $D$ and $f$ parameters

\begin{tabular}{|c|c|c|c|c|c|c|c|c|c|}
\hline \multirow[t]{2}{*}{$\overline{b \text { values }}$} & \multicolumn{3}{|c|}{ Low-perfusion (brain) } & \multicolumn{3}{|c|}{ Medium-perfusion (kidney) } & \multicolumn{3}{|c|}{ High-perfusion (liver) } \\
\hline & $\mathrm{SNR}=40$ & $\mathrm{SNR}=55$ & $\mathrm{SNR}=80$ & $\mathrm{SNR}=40$ & $\mathrm{SNR}=55$ & $\mathrm{SNR}=80$ & $\mathrm{SNR}=40$ & $\mathrm{SNR}=55$ & $\mathrm{SNR}=80$ \\
\hline$[200,1000]$ & $0.49 \pm 0.04$ & $0.47 \pm 0.05$ & $0.47 \pm 0.05$ & $0.22 \pm 0.05$ & $0.12 \pm 0.01$ & $0.12 \pm 0.01$ & $0.17 \pm 0.06$ & $\mathbf{0 . 0 3} \pm \mathbf{0 . 0 1}$ & $0.001 \pm 0.0005$ \\
\hline$[300,1000]$ & $0.32 \pm 0.02$ & $0.29 \pm 0.03$ & $0.29 \pm 0.03$ & $0.21 \pm 0.08$ & $\mathbf{0 . 0 5} \pm \mathbf{0 . 0 1}$ & $0.04 \pm 0.004$ & $0.21 \pm 0.08$ & $0.04 \pm 0.01$ & $0.001 \pm 0.0006$ \\
\hline$[400,1000]$ & $0.26 \pm 0.05$ & $0.18 \pm 0.02$ & $0.18 \pm 0.02$ & $0.26 \pm 0.10$ & $0.05 \pm 0.02$ & $0.01 \pm 0.001$ & $0.26 \pm 0.09$ & $0.05 \pm 0.02$ & $0.002 \pm 0.0007$ \\
\hline$[500,1000]$ & $0.25 \pm 0.08$ & $0.12 \pm 0.01$ & $0.11 \pm 0.01$ & $0.34 \pm 0.13$ & $0.06 \pm 0.02$ & $0.004 \pm 0.0004$ & $0.34 \pm 0.13$ & $0.06 \pm 0.02$ & $0.002 \pm 0.001$ \\
\hline$[600,1000]$ & $0.30 \pm 0.12$ & $0.09 \pm 0.01$ & $0.07 \pm 0.01$ & $0.45 \pm 0.16$ & $0.08 \pm 0.03$ & $0.003 \pm 0.002$ & $0.45 \pm 0.17$ & $0.08 \pm 0.3$ & $0.003 \pm 0.001$ \\
\hline$[700,1000]$ & $0.40 \pm 0.15$ & $0.09 \pm 0.03$ & $0.05 \pm 0.01$ & $0.61 \pm 0.19$ & $0.12 \pm 0.05$ & $0.004 \pm 0.002$ & $0.64 \pm 0.23$ & $0.12 \pm 0.05$ & $0.004 \pm 0.002$ \\
\hline$[800,1000]$ & $0.62 \pm 0.23$ & $0.13 \pm 0.06$ & $0.03 \pm 0.003$ & $0.92 \pm 0.33$ & $0.19 \pm 0.08$ & $0.007 \pm 0.003$ & $0.99 \pm 0.33$ & $0.19 \pm 0.08$ & $0.007 \pm 0.003$ \\
\hline$[900,1000]$ & $1.20 \pm 0.52$ & $0.26 \pm 0.12$ & $0.03 \pm 0.003$ & $1.78 \pm 0.74$ & $0.40 \pm 0.15$ & $0.01 \pm 0.007$ & $1.91 \pm 0.67$ & $0.41 \pm 0.16$ & $0.010 \pm 0.007$ \\
\hline
\end{tabular}

Lowest relative errors are highlighted in bold for each SNR level and perfusion model

The optimal $b$ value distributions are listed in Table 2 for each perfusion model. At SNR $=40$, the optimal $b$ value distributions were [500,1000], [300,1000], and [200,1000] for the low-, medium-, and high-perfusion models, respectively. The relative errors of the estimated $f$ parameters for these $b$ value distributions were $<20 \%$ at $\mathrm{SNR}=40$, and $<10 \%$ for SNR $=55$ and 80 . The $b$ value distribution for the low-perfusion model was higher because of the greater relative bias at the lower $b$ values.

\section{Volunteer data}

The $b$ value distributions [300,1000] and [500,1000] were investigated retrospectively for the volunteer 

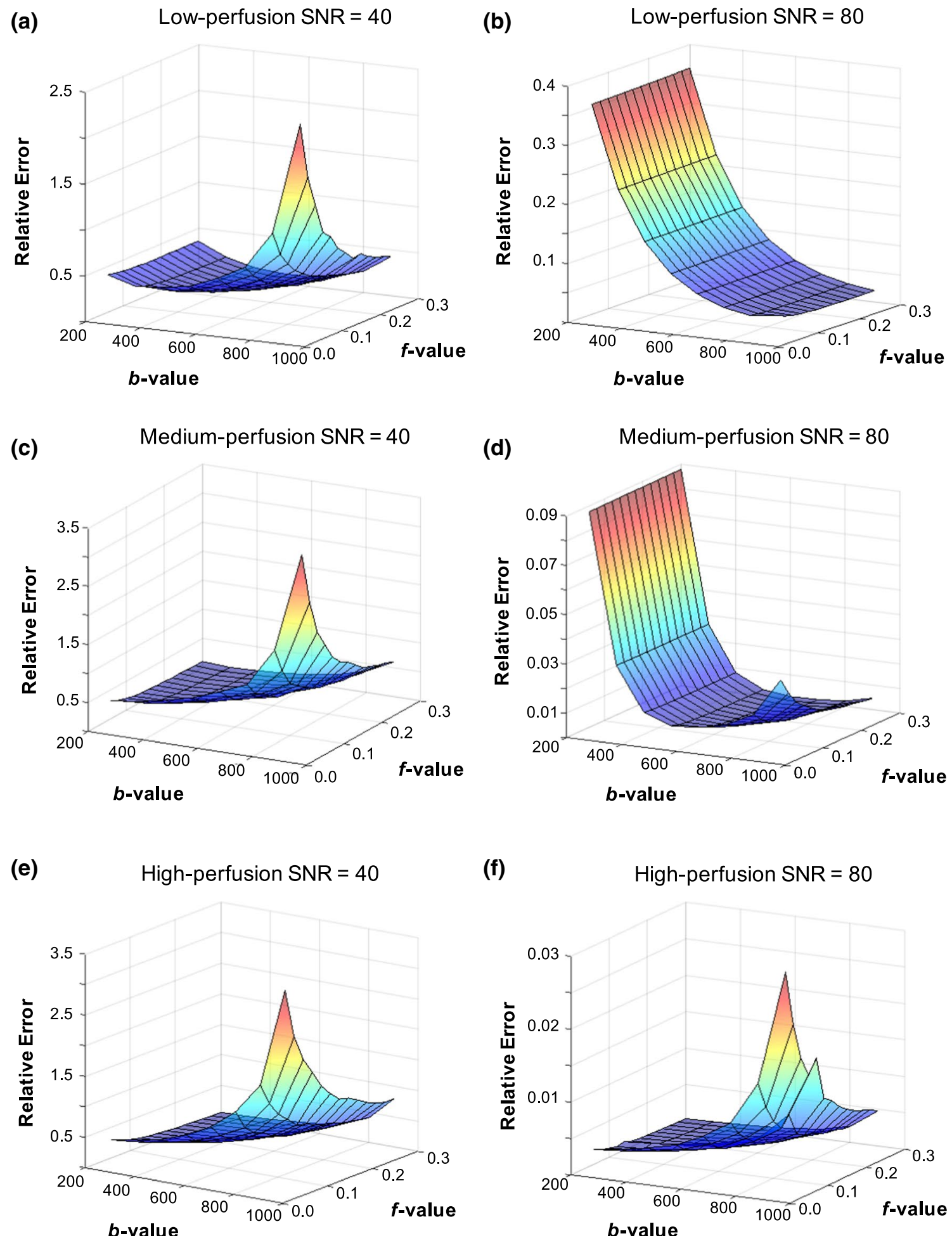

Fig. 5 Contour plots of the relative error of perfusion fraction, $f$, with different $b$ value distribution at $\mathrm{SNR}=40(\mathbf{a}, \mathbf{c}, \mathbf{e})$ and $\mathrm{SNR}=80(\mathbf{b}, \mathbf{d}, \mathbf{f})$ for the low- (a-b), medium- (c-d), and high-perfusion (e-f) models

cohort. The average values of $D$ and $f$ in the grey matter were $0.865 \pm 0.05\left(\times 10^{-3} \mathrm{~mm}^{2} / \mathrm{s}\right)$ and $0.141 \pm 0.02$ with [500,1000], and $0.912 \pm 0.05\left(\times 10^{-3} \mathrm{~mm}^{2} / \mathrm{s}\right)$ and $0.104 \pm 0.01$ with $[300,1000]$, respectively. The higher $f$ values and lower $D$ values derived with the $[500,1000]$ distribution agreed with the results from the low-perfusion model simulations. 
Table 2 Recommended $b$ value distributions for computation of IVIM perfusion fraction, based on relative error of $<10 \%$

\begin{tabular}{lllll}
\hline Model & SNR & $b$ value distribution & Relative error of $f(\%)$ & $\begin{array}{l}\text { Overall rela- } \\
\text { tive error (\%) }\end{array}$ \\
\hline Low-perfusion & 40 & {$[500,1000]$} & $18.7 \pm 5.5^{\mathrm{a}}$ & $24.8 \pm 7.6^{\mathrm{a}}$ \\
& 55 & {$[600,1000],[700,1000]$} & $<10$ & $<10$ \\
& 80 & {$[500,1000]$} & $<10$ & $<10$ \\
Medium-perfusion & 40 & {$[300,1000]$} & $15.3 \pm 8.3^{\mathrm{a}}$ & $21.3 \pm 7.8^{\mathrm{a}}$ \\
& 55 & {$[300,1000]$ to $[600,1000]$} & $<10$ & $<10$ \\
High-perfusion & 80 & $\geq[300,1000]$ & $<10$ & $<10$ \\
& 40 & {$[200,1000]$} & $12.1 \pm 6.8^{\mathrm{a}}$ & $17.2 \pm 6.4^{\mathrm{a}}$ \\
& 55 & {$[200,1000]$ to $[600,1000]$} & $<10$ & $<10$ \\
& 80 & {$[200,1000]$} & $<10$ & $<10$ \\
\hline
\end{tabular}

${ }^{\mathrm{a}}$ Lowest relative error
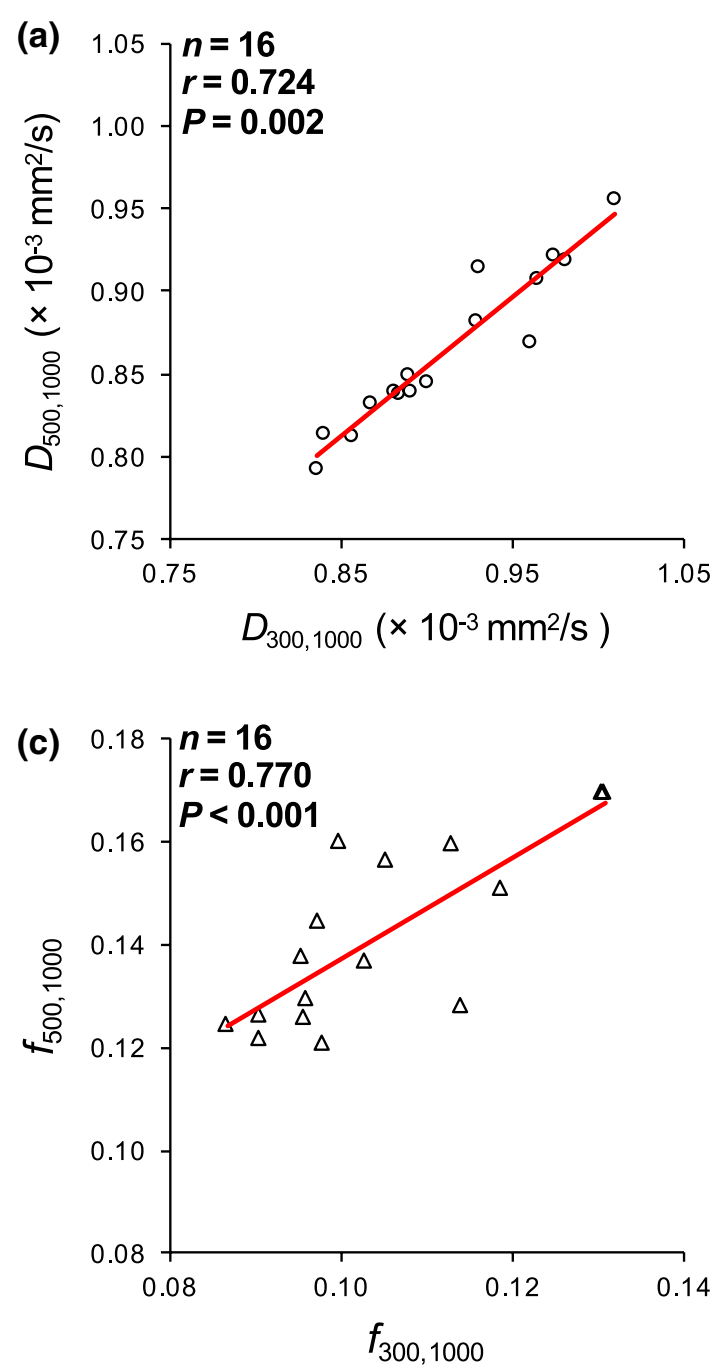

Fig. 6 Correlation $(\mathbf{a}, \mathbf{c})$ and Bland-Altman $(\mathbf{b}, \mathbf{d})$ plots for $D$ and $f$ parameters in grey matter with $b$ value distributions [500,1000] and $[300,1000]$ for the volunteer cohort $(n=16)$. The red lines in the BA
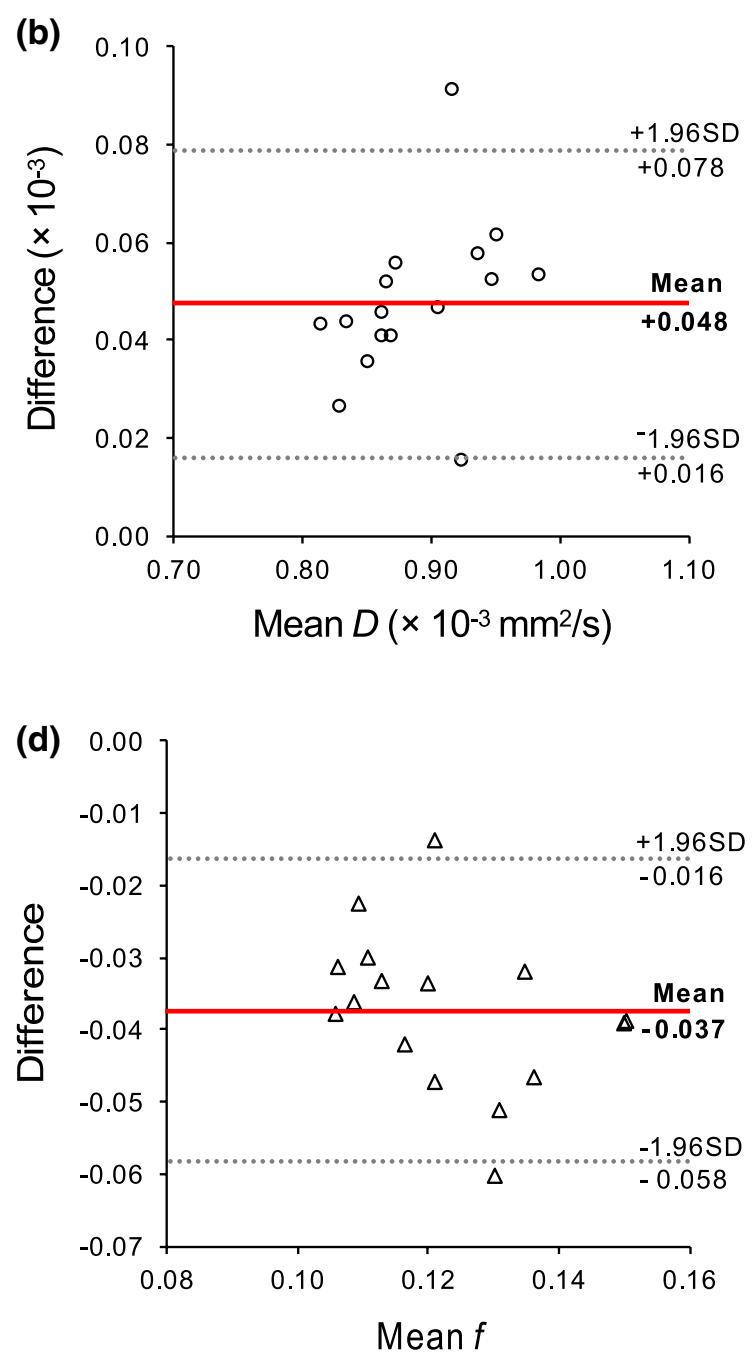

plots describe the mean difference of the values and the dashed lines the agreement range ( $95 \%$ confidence intervals) 
The correlation and Bland-Altman analysis for the estimated $D$ and $f$ parameters are presented in Fig. 6. Significant correlations were established between both IVIM parameters derived with the different $b$ value distributions, which indicated an existence of a linear relationship. Correlation of $r=0.724(P=0.002)$ was derived between the $D$ parameters, and $r=0.770(P<0.001)$ between the $f$ parameters. However, the estimation of $D(P=0.029)$ and $f(P<0.001)$ were significantly different between the $[500,1000]$ and $[300,1000]$ distributions. The agreement of methods, described by the Bland-Altman plots, showed a bias of $0.048\left(\times 10^{-3} \mathrm{~mm}^{2} / \mathrm{s}\right)$ and -0.037 for $D$ and $f$ parameters respectively. However, this only indicated the bias of estimating the IVIM parameters with $[300,1000]$ in comparison to $[500,1000]$. The bias was smaller at the lower $f$ values and greater towards the higher $f$ values. In comparison to the simulated $f=0.1$ value for the low-perfusion model at $\mathrm{SNR}=40$, the differences between the mean values for $[300,1000]$ and $[500,1000]$ were $0.015\left(\times 10^{-3} \mathrm{~mm}^{2} / \mathrm{s}\right)$ and -0.013 for $D$ and $f$ parameters, respectively. At $f=0.2$, the differences were increased to $0.030\left(\times 10^{-3} \mathrm{~mm}^{2} / \mathrm{s}\right)$ and -0.025 , although the relative bias remained the same.

The average grey matter histograms for the IVIM parameters are presented in Fig. 7, together with histograms for the low-perfused brain model (where $f=0.1$ at
SNR $=40$ ). Similar behaviour was observed between the in vivo and simulated data IVIM parameter histograms. The $f$ histogram based on the $[500,1000]$ distribution was shifted to higher $f$ values compared to the $[300,1000]$ distribution, with narrower distributions observed for the $[300,1000]$ distribution.

The ${ }_{\mathrm{w}} \mathrm{CV}$ was used to assess the repeatability of the IVIM parameters, which for the $[500,1000]$ and $[300,1000]$ distributions was 6.32 and $3.99 \%$ for $D$, and 15.3 and $10.8 \%$ for $f$, respectively. The values were similar to the ones depicted by the low-perfusion model at SNR $=40$ (Figs. 3, 4), with small improvements seen with the use of $[300,1000]$ over the $[500,1000]$ distribution.

The IVIM parameter histograms were compared to the ones derived with PVE masks for eight volunteer cases $(n=8)$. No significant differences were found between the mean, median, 10th and 90th percentiles of the IVIM parameters derived with the different masks for either [300,1000] or $[500,1000]$ distributions. Example grey matter masks and IVIM parameter maps derived with $b$ value distributions $[300,1000]$ and $[500,1000]$ are presented in Fig. 8 for a volunteer case. The overlaid regions on Fig. 8 a showed that the sulcal CSF was successfully removed with the binary grey matter mask. The $f$ maps derived with the $[300,1000]$ and $[500,1000]$ distributions were qualitatively similar, although
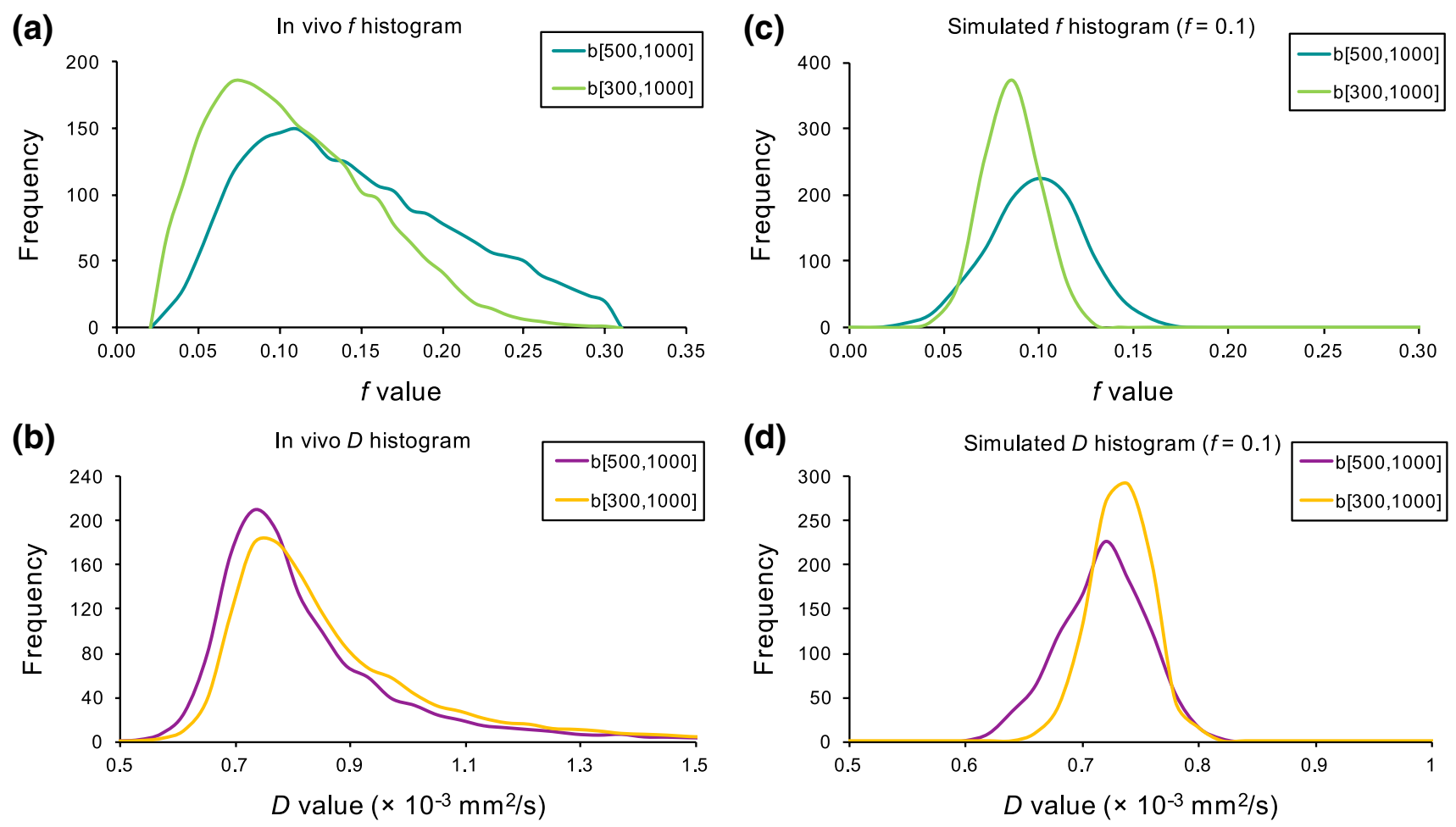

Fig. 7 Histograms of IVIM diffusion coefficient and perfusion fraction for in vivo (a-b) and simulated (c-d) data with $b$ value distributions $[500,1000]$ and $[300,1000]$. The in vivo histograms are the aver-

age histograms derived for the grey matter regions of the volunteer cohort and the simulated histograms correspond to the estimated values from the low-perfusion model at SNR $=40$ and $f=0.1$ 
Fig. 8 An example volunteer case with (a) $T_{1}$-weighted image and overlaid binary grey matter mask regions showing the exclusion of CSF, (b) the binary mask, (c) the PVE mask, and (d) the extracted IVIM $D$ (left) and $f$ (right) parameter maps derived with the $b$ value distributions [300,1000] and $[500,1000]$

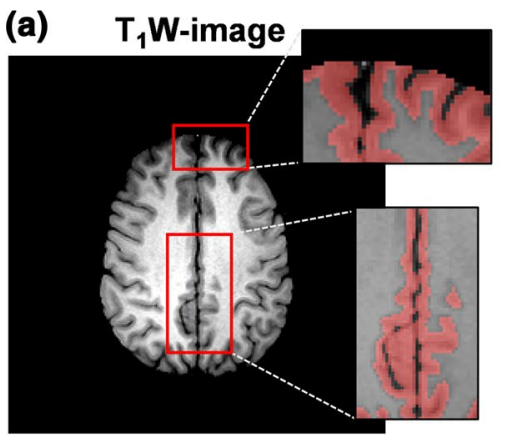

(b)

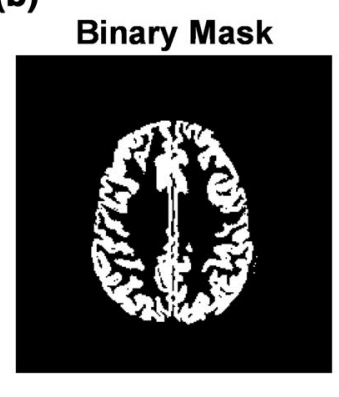

(c)

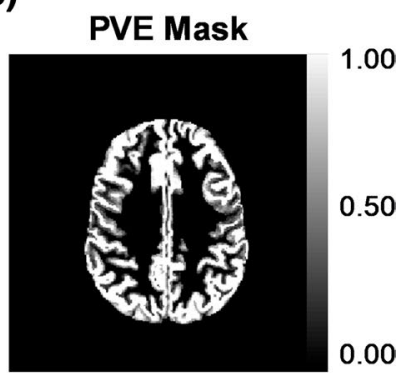

$[300,1000]$

(d)

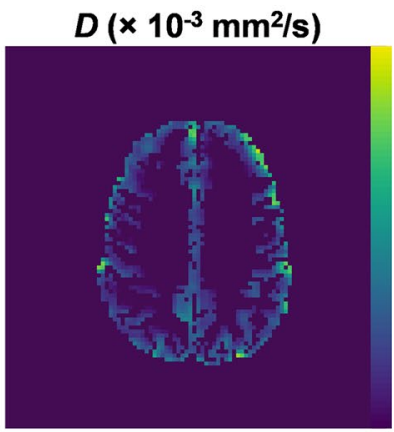

1.40

1.05

0.70

0.35

0.00

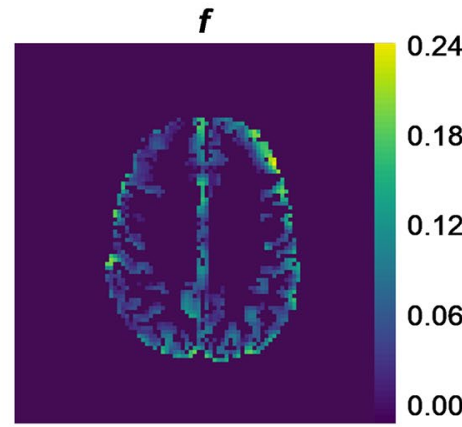

$D\left(\times 10^{-3} \mathrm{~mm}^{2} / \mathrm{s}\right)$

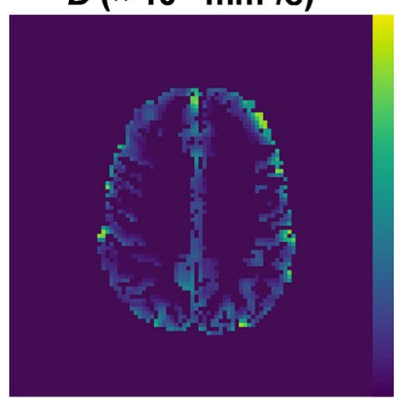

1.40

1.05

0.70
$[500,1000]$

differences in the magnitude of values could be observed, as depicted by the in vivo and simulation results.

\section{Discussion}

The use of a simple fitting approach with a minimum number of $b$ values was investigated to assess the feasibility of a rapid clinical application for determination of the IVIM perfusion fraction parameter. The accuracy and reliability of the IVIM parameters from different $b$ value distributions were assessed using model simulations and confirmed using the in vivo image data. The model data simulations demonstrated that the optimal $b$ value distributions for different tissue regions are dependent on the SNR level and the degree of perfusion influencing the diffusion signal.

The simulated tissue models were influenced by bias and noise to a different extent. Bias was found to be the dominant cause of higher relative errors at the low $D^{*} / D$ ratio and the low $b$ value distributions. A similar effect was seen in a study by Conklin et al. [18], who demonstrated a negative bias in estimating $f$ values in the brain by using a $b$ value distribution [300,900], compared to distributions including more intermediate $b$ values. The other cause for the higher relative errors was noise, which affected the estimation of perfusion fraction at higher $b$ value distributions. This resulted in greater variability in extrapolating the linear fit back to the $y$-axis from the high $b$ values. For the low-perfusion model, representing tissue perfusion of the brain, the effects of bias and noise were found to be minimised with the use of $b$ value distribution $[500,1000]$. The intrinsic bias seen at the low perfusion meant that also at the higher SNR levels the recommended $b$ value distribution was $\geq[500,1000]$.

The in vivo brain results indicated similar characteristics in the estimation of IVIM parameters as observed with the simulated model data. Higher $f$ values were estimated with the $[500,1000]$ distribution in comparison to the $[300,1000]$ distribution, though the bias between the two $b$ value distributions was higher in vivo compared to the simulated values where $f=0.1$. However, this was expected with the 
variations observed in the $D * / D$ ratio in vivo. The contributions from any potential partial volume effects due to the presence of CSF were minimised with the exclusion of sulcal CSF. This was confirmed by the comparison of IVIM values derived with the binary and PVE masks, which showed no difference in the distribution of the extracted IVIM values. The PVE masks were strictly generated and no voxels presenting tissue but grey matter were included. Nonetheless, the in vivo bias was within the observed range for the simulated $f$ values $(0.06-0.3)$ for the low-perfusion model, and confirms the presence of bias in low-perfused brain tissues. Therefore, consideration should be given to the impact of bias when choosing the $b$ value distribution for the IVIM analysis, in particular for low-perfused tissues such as the brain or breast $[3,29,30]$.

The medium- and high-perfusion models, representing tissues found in the abdomen, resulted in a substantial decrease in bias of estimating the perfusion fraction. Therefore, the more important factor for the optimal $b$ value distribution was the variability arising from the noise. The estimation of $f$ was found to be more sensitive to noise in comparison to $D$, although the increase in SNR level improved the reproducibility of both parameters considerably. The smaller contribution from bias meant that the lower $b$ value distributions had lower relative errors in contrast to the low-perfusion model, with the optimal $b$ value distributions for the medium-and high-perfusion models suggested to be $[300,1000]$ and $[200,1000]$, respectively.

The recommended $b$ values from this study can be used to inform analysis of pre-existing data of different tissue types. The constrained fitting approach uses a $b$ value threshold for the first fitting step on evaluating $D$ and $f$, where perfusion effects are assumed to be negligible. Previously, thresholds of $b=100 \mathrm{~s} / \mathrm{mm}^{2}$ for abdominal organs [31] and $200 \mathrm{~s} / \mathrm{mm}^{2}$ for the brain [23] have been suggested, when using the constrained fitting. In our study, the use of $b$ values $<500 \mathrm{~s} /$ $\mathrm{mm}^{2}$ for the low-perfusion model demonstrated high biases in estimation of $D$ and $f$ parameters, resulting in higher inaccuracies for any relatively low-perfused region. For higher perfused tissues, such as seen for the abdominal organs, the use of a lower $b$ value threshold is reasonable due to the lower influence of bias. Although the $b$ value recommendations were based on a relatively simple method of combining $D$ and $f$ errors, the aim was to provide $b$ values that can guide the choice of $b$ values, and minimise the intrinsic bias that arises from the fitting, even when using high quality data.

Previously reported IVIM parameters for different pathologies are listed in Table 3. For highly perfused tissues, such as reported for cirrhotic liver $[8,9,32]$ hepatocellular carcinomas $[5,33]$, prostate cancer $[34,35]$ and many of the pancreas related pathologies [12, 36, 37], our results suggest that the use of a low $b$ value can reduce the variability in estimating the perfusion fraction. In lower perfused tissues, such as reported for breast cancer [3, 29, 30], a higher $b$ value can aid to reduce the bias.

The other low perfused region of clinical interest is the brain. Previous IVIM studies of brain gliomas have been inconclusive with the reported $f$ values $[4,17,38,39]$. A range of values were reported for low- $\left(D^{*} 2.15-11.4 \times 10^{-3}\right.$ $\left.\mathrm{mm}^{2} / \mathrm{s}, f 0.06-0.49\right)$ and high-grade $\left(D^{*} 2.7-41.610^{-3}\right.$ $\left.\mathrm{mm}^{2} / \mathrm{s}, f 0.11-0.40\right)$ gliomas. Interestingly, the two studies [17, 39] including $b$ values $\geq 1500$ and up to $3500 \mathrm{~s} /$ $\mathrm{mm}^{2}$, reported relatively high $f$ values for the brain $(\geq 0.29)$, whereas the studies including $b$ values $\leq 1300 \mathrm{~s} / \mathrm{mm}^{2}[4$, 38 ] reported much lower values $(\leq 0.13)$. Tri-exponential fitting has been previously used for data with high $b$ values $\left(>1000 \mathrm{~s} / \mathrm{mm}^{2}\right)$ in the brain [40], suggesting that using a bi-exponential fitting for higher $b$ value data might result in under fitting and thus potential positive bias in estimation of the IVIM parameters. On the other hand, both the IVIM model and the tri-exponential model are unable to account for the non-Gaussian diffusion and noise observed at high $b$ values [41]. An alternative method was introduced with the use of the IVIM kurtosis model, which can fully account for the non-Gaussian behaviour, as shown previously in a study by Iima et al. [29] investigating low-perfused breast tissue up to $b$ values $=2500 \mathrm{~s} / \mathrm{mm}^{2}$. Other challenges at the higher $b$ values include the SNR level, which can be relatively low, and consequently increases the variability of the data, if not adjusted e.g. with the use of higher NSA. In the context of these issues, the use of the standard IVIM model at $b$ values above $1000 \mathrm{~s} / \mathrm{mm}^{2}$ might not be desirable. Overall, the differences in these studies make it challenging to assess the accuracy of the reported IVIM values, and therefore for studies in the relatively low perfused tissues, an estimate of the SNR level should be of importance as well as caution in the use of lower and higher $b$ values, which can introduce bias to the results.

The increase of SNR by the increase in number of signal averages provided great improvements in the reliability of the estimated IVIM parameters. The increase from $\mathrm{SNR}=40$ to $\mathrm{SNR}=55$, corresponding to approximately an acquisition with one and two signal averages, increased the reproducibility for all the $b$ value distributions. The improvement was less marked in going from an SNR $=55-80$. Therefore, aiming for an SNR = 55 may be a reasonable compromise between reproducibility and length of acquisition, if the biological effects being investigated are large enough, such as seen between the low- and high-grade gliomas. Presence of small biological changes in tissue might require the use of higher SNR levels, where detection of the tissue properties can be improved with the better reproducibility of the $f$ parameter.

Optimisation of $b$ values for specific tissue regions with specific fitting methods have been reported previously $[12,42,43]$. The results from these studies include the $D^{*}$ 
Table 3 Previous IVIM studies of different pathologies and the reported IVIM parameters

\begin{tabular}{|c|c|c|c|c|c|}
\hline Study & Pathology & No. of patients & $D^{* / D}$ & $D^{*}$ & $f$ \\
\hline \multirow[t]{2}{*}{ Bisdas et al. [38] } & Low-grade glioma & 7 & 20.8 & 10.8 & 0.06 \\
\hline & High-grade glioma & 15 & 54.7 & 41.6 & 0.11 \\
\hline \multirow[t]{2}{*}{ Federau et al. [4] } & Low-grade glioma & 5 & 11.4 & 11.4 & 0.08 \\
\hline & High-grade glioma & 16 & 5.85 & 11.7 & 0.13 \\
\hline \multirow[t]{2}{*}{ Hu et al. [17] } & Low-grade glioma & 13 & 2.84 & 2.15 & 0.48 \\
\hline & High-grade glioma & 29 & 5.35 & 2.71 & 0.29 \\
\hline \multirow[t]{2}{*}{ Lin et al. [39] } & Low-grade glioma & 13 & 24.7 & 2.77 & 0.49 \\
\hline & High-grade glioma & 11 & 29.0 & 5.10 & 0.40 \\
\hline Suo et al. [6] & Ischemic stroke & 101 & 24.3 & 10.2 & 0.04 \\
\hline Cho et al. [3] & Breast cancer & 14 & 10.7 & 15.0 & 0.13 \\
\hline Sigmund et al. [30] & Breast cancer & 27 & 6.40 & 15.1 & 0.10 \\
\hline Hayashi et al. [32] & Cirrhotic liver & 29 & 28.7 & 25.0 & 0.24 \\
\hline Luciani et al. [8] & Cirrhotic liver & 12 & 51.2 & 61.0 & 0.30 \\
\hline Patel et al. [9] & Cirrhotic liver & 14 & 26.8 & 27.9 & 0.25 \\
\hline Kuru et al. [34] & Prostate cancer & 27 & 29.9 & 31.1 & 0.10 \\
\hline Ueda et al. [35] & Prostate cancer & 63 & 11.5 & 7.48 & 0.23 \\
\hline Hectors et al. [5] & Hepatocellular carcinoma & 25 & 47.1 & 64.1 & 0.18 \\
\hline \multirow[t]{2}{*}{ Woo et al. [33] } & Low-grade hepatocellular carcinoma & 24 & 31.1 & 36.6 & 0.22 \\
\hline & High-grade hepatocellular carcinoma & 18 & 32.5 & 32.3 & 0.19 \\
\hline Lemke et al. [12] & Pancreatic adenocarcinoma & 23 & $17.4^{\mathrm{a}}$ & $20.0^{\mathrm{a}}$ & 0.09 \\
\hline \multirow[t]{4}{*}{ Kang et al. [36] } & Chronic pancreatitis & 7 & 28.9 & 40.8 & 0.19 \\
\hline & Neuroendocrine tumour & 17 & 39.4 & 43.7 & 0.30 \\
\hline & Pancreatic adenocarcinoma & 39 & 19.6 & 22.3 & 0.12 \\
\hline & Intraductal papillary mucinous neoplasm & 37 & 5.49 & 15.6 & 0.10 \\
\hline \multirow[t]{2}{*}{ Klauss et al. [37] } & Chronic pancreatitis & 9 & $18.7^{\mathrm{a}}$ & $20.0^{\mathrm{a}}$ & 0.16 \\
\hline & Pancreatic adenocarcinoma & 15 & $18.7^{\mathrm{a}}$ & $20.0^{\mathrm{a}}$ & 0.08 \\
\hline
\end{tabular}

${ }^{\mathrm{a}} D^{*}$ fixed at $20 \times 10^{-3} \mathrm{~mm}^{2} / \mathrm{s}$ parameter in the computation of the overall errors, which means that most of the contribution is likely to come from the $D^{*}$ due to its poor reliability $[12,13,44]$. This results in optimised $D^{*}$ parameter, but the variation of $f$ might not have been taken into consideration. In our study, only the $D$ and $f$ parameters were considered, with larger contribution coming from the relative error of the $f$ parameter. The recent interest in the $f$ parameter for various brain pathologies, as well as for many types of cancer, indicates that a simple, but reliable approach is required for the transfer of IVIM to clinical imaging $[45,46]$.

The most used diffusion parameter in clinical practice remains the apparent diffusion coefficient (ADC). However, the use of $D$ has shown better diagnostic performance in comparison to ADC in recent studies [33, 47]. Therefore, a clinical protocol with three $b$ values could provide the option for computation of ADC, as well as the IVIM parameters $D$ and $f$. The method used in this study can be easily adapted for clinical use by the introduction of a $b$ value to an already routine protocol with $b$ values 0 and 1000 with a small cost in scan duration. However, as suggested by the model simulations, awareness of the image quality and hence SNR is critical for the assessment of reliability of the derived IVIM parameters. Additional stability in the fitting of IVIM parameters can be achieved by increasing the number of averages, which were shown to provide large improvements on the results.

This study had some limitations. First, only three separate tissue models were investigated. While this provides a general guide on the use of optimal $b$ values, variation in tissues creates a more complex scenario as indicated by the larger differences seen in vivo in comparison to the simulated results. Pathologies in the abdomen and the surrounding tissue have been found relative high perfused, implying that the recommended $b$ value is likely to perform well for the whole imaged region. However, imaging in the abdomen can be affected by respiratory and cardiac motions, which must be assessed to ensure sufficient image quality for IVIM analysis. The $b$ value choice for the brain is more complex, where bias is likely to play a greater role, and therefore, the use of higher $b$ values should be considered. A second limitation is the importance of the noise level for the selection of $b$ values. As with any imaging modality, data quality is important and an estimate of the SNR level can provide 
a good guidance on the reliability of the results and aid in choosing the optimal $b$ values. Finally, a limitation of this study is the lack of availability of software for use in clinical practice, which is currently not offered on clinical workstations.

\section{Conclusion}

This study demonstrated that IVIM parameters $D$ and $f$ can be estimated reliably with three $b$ values. We have shown using model simulations that the optimal $b$ value distribution depends on the diffusion and perfusion characteristics of the tissue and the compromise between bias and variability, which were validated using in vivo IVIM measurements. Recommendations for $b$ values were made based on the model simulations, which can be used as a guide in future studies or for pre-existing data. With different clinical centres utilising different $b$ value distributions, the results from this study can also aid in interpretation of differences seen between IVIM parameters of similar tissues.

Acknowledgements This work was funded by the Engineering and Physical Sciences Research Council (EPSRC, EP/F50053X/1), the National Institute for Health Research (NIHR) via a Research Professorship (13-0053), and Free Radio in conjunction with Help Harry Help Others (HHHO).

Author contributions EMM: project development, data management and data analysis. JN: project development, data analysis. HD: project development. ACP: project development, data analysis.

\section{Compliance with ethical standards}

Conflict of interest The authors declare that they have no conflict of interest.

Ethical approval This study was approved by the East Midlands - Derby Research Ethics Committee (REC04/MRE04/41), UK. All procedures performed in studies involving human participants were in accordance with the ethical standards of the institutional and/or national research committee and with the 1975 Helsinki declaration and its later amendments or comparable ethical standards.

Open Access This article is distributed under the terms of the Creative Commons Attribution 4.0 International License (http://creativecommons.org/licenses/by/4.0/), which permits unrestricted use, distribution, and reproduction in any medium, provided you give appropriate credit to the original author(s) and the source, provide a link to the Creative Commons license, and indicate if changes were made.

\section{References}

1. Le Bihan D, Breton E, Lallemand D, Aubin ML, Vignaud J, LavalJeantet M (1988) Separation of diffusion and perfusion in intravoxel incoherent motion MR imaging. Radiology 168(2):497-505
2. Le Bihan D, Breton E, Lallemand D, Grenier P, Cabanis E, LavalJeantet M (1986) MR imaging of intravoxel incoherent motions: application to diffusion and perfusion in neurologic disorders. Radiology 161(2):401-407

3. Cho GY, Moy L, Zhang JL, Baete S, Lattanzi R, Moccaldi M, Babb JS, Kim S, Sodickson DK, Sigmund EE (2015) Comparison of fitting methods and $b$-value sampling strategies for intravoxel incoherent motion in breast cancer. Magn Reson Med 74(4):1077-1085

4. Federau C, Meuli R, O’Brien K, Maeder P, Hagmann P (2014) Perfusion measurement in brain gliomas with intravoxel incoherent motion MRI. Am J Neuroradiol 35(2):256-262

5. Hectors SJ, Wagner M, Besa C, Bane O, Dyvorne HA, Fiel MI, Zhu H, Donovan M, Taouli B (2016) Intravoxel incoherent motion diffusion-weighted imaging of hepatocellular carcinoma: is there a correlation with flow and perfusion metrics obtained with dynamic contrast-enhanced MRI? J Magn Reson Imaging 44(4):856-864

6. Suo S, Cao M, Zhu W, Li L, Li J, Shen F, Zu J, Zhou Z, Zhuang Z, Qu J, Chen Z, Xu J (2016) Stroke assessment with intravoxel incoherent motion diffusion-weighted MRI. NMR Biomed 29(3):320-328

7. Wirestam R, Brockstedt S, Lindgren A, Geijer B, Thomsen C, Holtas S, Stahlberg F (1997) The perfusion fraction in volunteers and in patients with ischaemic stroke. Acta Radiol 38(6):961-964

8. Luciani A, Vignaud A, Cavet M, Van Nhieu JT, Mallat A, Ruel L, Laurent A, Deux JF, Brugieres P, Rahmouni A (2008) Liver cirrhosis: intravoxel incoherent motion MR imaging-pilot study. Radiology 249(3):891-899

9. Patel J, Sigmund EE, Rusinek H, Oei M, Babb JS, Taouli B (2010) Diagnosis of cirrhosis with intravoxel incoherent motion diffusion MRI and dynamic contrast-enhanced MRI alone and in combination: preliminary experience. J Magn Reson Imaging 31(3):589-600

10. Barbieri S, Donati OF, Froehlich JM, Thoeny HC (2016) Impact of the calculation algorithm on biexponential fitting of diffusionweighted MRI in upper abdominal organs. Magn Reson Med 75(5):2175-2184

11. Cohen AD, Schieke MC, Hohenwalter MD, Schmainda KM (2015) The effect of low b-values on the intravoxel incoherent motion derived pseudodiffusion parameter in liver. Magn Reson Med 73(1):306-311

12. Lemke A, Stieltjes B, Schad LR, Laun FB (2011) Toward an optimal distribution of $b$ values for intravoxel incoherent motion imaging. Magn Reson Imaging 29(6):766-776

13. Meeus EM, Novak J, Withey SB, Zarinabad N, Dehghani H, Peet AC (2017) Evaluation of intravoxel incoherent motion fitting methods in low-perfused tissue. J Magn Reson Imaging 45(5):1325-1334

14. Grech-Sollars M, Hales PW, Miyazaki K, Raschke F, Rodriguez D, Wilson M, Gill SK, Banks T, Saunders DE, Clayden JD, Gwilliam MN, Barrick TR, Morgan PS, Davies NP, Rossiter J, Auer DP, Grundy R, Leach MO, Howe FA, Peet AC, Clark CA (2015) Multi-centre reproducibility of diffusion MRI parameters for clinical sequences in the brain. NMR Biomed 28(4):468-485

15. Schneider MJ, Dietrich O, Ingrisch M, Helck A, Winter KS, Reiser MF, Staehler M, Casuscelli J, Notohamiprodjo M (2016) Intravoxel incoherent motion magnetic resonance imaging in partially nephrectomized kidneys. Invest Radiol 51(5):323-330

16. Federau C, O’Brien K, Meuli R, Hagmann P, Maeder P (2014) Measuring brain perfusion with intravoxel incoherent motion (IVIM): initial clinical experience. J Magn Reson Imaging 39(3):624-632

17. Hu YC, Yan LF, Wu L, Du P, Chen BY, Wang L, Wang SM, Han Y, Tian Q, Yu Y, Xu TY, Wang W, Cui GB (2014) Intravoxel 
incoherent motion diffusion-weighted MR imaging of gliomas: efficacy in preoperative grading. Sci Rep 4:7208

18. Conklin J, Heyn C, Roux M, Cerny M, Wintermark M, Federau C (2016) A simplified model for intravoxel incoherent motion perfusion imaging of the brain. AJNR Am J Neuroradiol 37(12):2251-2257

19. Park HJ, Sung YS, Lee SS, Lee Y, Cheong H, Kim YJ, Lee MG (2016) Intravoxel incoherent motion diffusion-weighted MRI of the abdomen: the effect of fitting algorithms on the accuracy and reliability of the parameters. J Magn Reson Imaging. doi:10.1002/ jmri.25535

20. Li YT, Cercueil JP, Yuan J, Chen W, Loffroy R, Wáng YXJ (2017) Liver intravoxel incoherent motion (IVIM) magnetic resonance imaging: a comprehensive review of published data on normal values and applications for fibrosis and tumor evaluation. Quantitative Imaging in Medicine and Surgery 7(1):59-78

21. Wu WC, Chen YF, Tseng HM, Yang SC, My PC (2015) Caveat of measuring perfusion indexes using intravoxel incoherent motion magnetic resonance imaging in the human brain. Eur Radiol 25(8):2485-2492

22. Gudbjartsson H, Patz S (1995) The rician distribution of noisy MRI data. Magn Reson Med 34(6):910-914

23. Le Bihan D, Turner R, MacFall JR (1989) Effects of intravoxel incoherent motions (IVIM) in steady-state free precession (SSFP) imaging: application to molecular diffusion imaging. Magn Reson Med 10(3):324-337

24. (NEMA) NEMA (2001) Determination of signal-to-noise ratio (SNR) in diagnostic magnetic resonance imaging. National electrical manufacturers association

25. Smith SM, Jenkinson M, Woolrich MW, Beckmann CF, Behrens TE, Johansen-Berg H, Bannister PR, De Luca M, Drobnjak I, Flitney DE, Niazy RK, Saunders J, Vickers J, Zhang Y, De Stefano N, Brady JM, Matthews PM (2004) Advances in functional and structural MR image analysis and implementation as FSL. Neuroimage 23(Suppl 1):S208-219

26. Zhang Y, Brady M, Smith S (2001) Segmentation of brain MR images through a hidden Markov random field model and the expectation-maximization algorithm. IEEE Trans Med Imaging 20(1):45-57

27. Barnhart HX, Barboriak DP (2009) Applications of the repeatability of quantitative imaging biomarkers: a review of statistical analysis of repeat data sets. Transl Oncol 2(4):231-235

28. Hyslop NP, White WH (2009) Estimating precision using duplicate measurements. J Air Waste Manag Assoc 59(9):1032-1039

29. Iima M, Yano K, Kataoka M, Umehana M, Murata K, Kanao S, Togashi K, Le Bihan D (2015) Quantitative non-Gaussian diffusion and intravoxel incoherent motion magnetic resonance imaging: differentiation of malignant and benign breast lesions. Invest Radiol 50(4):205-211

30. Sigmund EE, Cho GY, Kim S, Finn M, Moccaldi M, Jensen JH, Sodickson DK, Goldberg JD, Formenti S, Moy L (2011) Intravoxel incoherent motion imaging of tumor microenvironment in locally advanced breast cancer. Magn Reson Med 65(5):1437-1447

31. Taouli B, Beer AJ, Chenevert T, Collins D, Lehman C, Matos C, Padhani AR, Rosenkrantz AB, Shukla-Dave A, Sigmund E, Tanenbaum L, Thoeny H, Thomassin-Naggara I, Barbieri S, Corcuera-Solano I, Orton M, Partridge SC, Koh DM (2016) Diffusion-weighted imaging outside the brain: consensus statement from an ISMRM-sponsored workshop. J Magn Reson Imaging 44(3):521-540
32. Hayashi T, Miyati T, Takahashi J, Fukuzawa K, Sakai H, Tano M, Saitoh S (2013) Diffusion analysis with triexponential function in liver cirrhosis. J Magn Reson Imaging 38(1):148-153

33. Woo S, Lee JM, Yoon JH, Joo I, Han JK, Choi BI (2014) Intravoxel incoherent motion diffusion-weighted MR imaging of hepatocellular carcinoma: correlation with enhancement degree and histologic grade. Radiology 270(3):758-767

34. Kuru TH, Roethke MC, Stieltjes B, Maier-Hein K, Schlemmer HP, Hadaschik BA, Fenchel M (2014) Intravoxel incoherent motion (IVIM) diffusion imaging in prostate cancer-what does it add? J Comput Assist Tomogr 38(4):558-564

35. Ueda Y, Takahashi S, Ohno N, Kyotani K, Kawamitu H, Miyati T, Aoyama N, Ueno Y, Kitajima K, Kawakami F, Okuaki T, Tsukamoto R, Yanagita E, Sugimura K (2016) Triexponential function analysis of diffusion-weighted MRI for diagnosing prostate cancer. J Magn Reson Imaging 43(1):138-148

36. Kang KM, Lee JM, Yoon JH, Kiefer B, Han JK, Choi BI (2014) Intravoxel incoherent motion diffusion-weighted MR imaging for characterization of focal pancreatic lesions. Radiology 270(2):444-453

37. Klauss M, Lemke A, Grunberg K, Simon D, Re TJ, Wente MN, Laun FB, Kauczor HU, Delorme S, Grenacher L, Stieltjes B (2011) Intravoxel incoherent motion MRI for the differentiation between mass forming chronic pancreatitis and pancreatic carcinoma. Invest Radiol 46(1):57-63

38. Bisdas S, Koh TS, Roder C, Braun C, Schittenhelm J, Ernemann U, Klose U (2013) Intravoxel incoherent motion diffusionweighted MR imaging of gliomas: feasibility of the method and initial results. Neuroradiology 55(10):1189-1196

39. Lin YK, Li JR, Zhang ZQ, Xu Q, Zhou ZY, Zhang ZP, Zhang Y, Zhang ZJ (2015) Comparison of intravoxel incoherent motion diffusion-weighted $\mathrm{mr}$ imaging and arterial spin labeling MR imaging in gliomas. Biomed Res Int. doi:10.1155/2015/234245

40. Ohno N, Miyati T, Kobayashi S, Gabata T (2016) Modified triexponential analysis of intravoxel incoherent motion for brain perfusion and diffusion. J Magn Reson Imaging 43(4):818-823

41. Yablonskiy DA, Sukstanskii AL (2010) Theoretical models of the diffusion weighted MR signal. NMR Biomed 23(7):661-681

42. Dyvorne H, Jajamovich G, Kakite S, Kuehn B, Taouli B (2014) Intravoxel incoherent motion diffusion imaging of the liver: optimal b-value subsampling and impact on parameter precision and reproducibility. Eur J Radiol 83(12):2109-2113

43. Zhang JL, Sigmund EE, Rusinek H, Chandarana H, Storey P, Chen Q, Lee VS (2012) Optimization of b-value sampling for diffusionweighted imaging of the kidney. Magn Reson Med 67(1):89-97

44. Lee Y, Lee SS, Kim N, Kim E, Kim YJ, Yun SC, Kuhn B, Kim IS, Park SH, Kim SY, Lee MG (2015) Intravoxel incoherent motion diffusion-weighted MR imaging of the liver: effect of triggering methods on regional variability and measurement repeatability of quantitative parameters. Radiology 274(2):405-415

45. Iima M, Le Bihan D (2016) Clinical intravoxel incoherent motion and diffusion MR imaging: past, present, and future. Radiology 278(1):13-32

46. Le Bihan D (2008) Intravoxel incoherent motion perfusion MR imaging: a wake-up call. Radiology 249(3):748-752

47. Chandarana H, Kang SK, Wong S, Rusinek H, Zhang JL, Arizono S, Huang WC, Melamed J, Babb JS, Suan EF, Lee VS, Sigmund EE (2012) Diffusion-weighted intravoxel incoherent motion imaging of renal tumors with histopathologic correlation. Invest Radiol 47(12):688-696 